\title{
EFFECTS OF ANXIETY AND MORPHINE ON DISCRIMINATION OF INTENSITIES OF PAINFUL STIMULI
}

\author{
BY HARRIS E. HILL, CONAN H. KORNETSKY, HAROLD G. FLANARY, AND \\ ABRAHAM WIKLER \\ (From the National Institute of Mental Health, Addiction Research Center, Public Health \\ Service Hospital, Lexington, $K y$.)
}

(Submitted for publication January 10, 1952; accepted March 17, 1952)

\section{INTRODUCTION}

The testing of analgesics, especially morphine, has yielded a great deal of conflicting evidence concerning the effects of drugs on pain perception. The recent reviews by Wikler (1) and Edwards (2), and the monograph by Wolff and Wolf (3), cover thoroughly the studies that have attempted to measure changes in the pain perception threshold. Although many investigators have reported rises in the pain perception threshold of some of their subjects following the administration of morphine, the majority have obtained variable results. Wolff, Hardy, and Goodell (4) found consistent rises following the administration of morphine under certain conditions. Using the same technique, Andrews (5), Isbell (6), Denton and Beecher (7), and Chapman and Jones (8) found, following morphine, that the pain perception threshold might be elevated, lowered or unchanged. Similar results were obtained by Isbell and Frank (9) in studies on the effect of analgesics on tooth pain perception thresholds.

It would seem that the discrepancies were not due to faulty apparatus or lack of objectivity in handling the data. Rather, the reasons appear to lie in conditions that were not held constant or that were beyond the control of the experimenters. One such variable that has been mentioned in several studies as possibly contributing to unpredictability of results is the "emotional" status of the subject at the time the experiment is performed. Included would be such factors as response of the subject to the experimental room, to the complex apparatus, to the attitude of the experimenter, and to the expected painful stimuli. The uncontrolled variable in the subject's behavior would then seem to comprise that class of responses which is termed anticipatory, i.e., especially affectively toned responses that are anticipatory of pain. This, of course, is one type of fear or "anxiety."
Isbell and Frank (9) also studied the effect of morphine on the ability of subjects to estimate intensities of painful stimuli. In their investigation, the pulp nerve of a tooth containing a silver amalgam filling was stimulated electrically at intensities three times the absolute perception threshold value. These stimuli, which served as standards, were definitely painful. After morphine the subjects were required to manipulate rheostat knobs on the stimulating apparatus until the test stimuli were reported as being equal to the standard. (The apparatus was so adjusted that turns of the knob did not correspond with the intensity of stimuli or with dial scale readings.) No change in the accuracy of estimation of painful stimuli was observed following the administration of morphine. However, analysis of the conditions under which the studies were conducted indicates that considerable effort was expended to reduce anxiety in the subjects; they were given considerable control of the experimental situation since they manipulated the stimulator knob themselves and applied the electrode to their own tooth fillings. In addition they were trained in estimating intensities of painful stimuli for approximately one week prior to testing. In view of conditions and results discussed in published studies on pain perception thresholds, it might be expected that if conditions had been favorable for enhancement of anxiety (anticipatory responses to expected unpleasant stimuli), Isbell and Frank would have obtained different results.

The present investigation is an attempt to determine the extent to which anxiety and morphine alter pain intensity estimation. The experiment was designed to investigate the effect of controlled variations in the experimenter's treatment of the subject on estimation of pain intensities, while the number, the order, and the intensity of the stimuli were kept constant for all groups. If it could be 
demonstrated that morphine alters pain intensity estimation under the usual experimental conditions and produces no comparable alteration under circumstances designed to alleviate anxiety, it would be very strongly indicated that morphine exerts a significant influence upon pain intensity estimation only when anticipation of pain is present. The conclusion would then be apparent that failure to consider the variable of anticipation might be responsible for the reported variations in pain perception thresholds following the administration of analgesics.

Specifically, the present report deals with the effects of single analgesic doses of morphine (15 mg. subcutaneously) on the ability of subjects to compare intensities of painful stimuli under two conditions: (1) proceeding with the experiment in the usual way without familiarizing the subject with the potentially "fear-inspiring" experimental situation; and (2) preceding and accompanying experimentation with measures designed to allay the subject's anxiety. As indicated, it was hypothesized $(a)$ that overestimation of intensity would occur under condition (1), (b) that morphine would significantly reduce this overestimation, (c) that little overestimation of the stimuli would occur under condition (2), and (d) that morphine would exert no significant impairment of estimation under condition (2).

\section{METHODS}

Subjects. Forty-two white and colored post-addicts (individuals with previous histories of opiate addiction who had not received such drugs for a period of several months prior to the experiment) acted as subjects. They were unselected except that several prospective subjects were disqualified, some because of ill-health, others because of refusal to complete the test, and others because of difficulties with apparatus at the time of testing. Each subject served two days, the sessions being separated by 48 hours. Half of each group received medication on the first day and the other half received it on the second day. Assignment to particular groups was made a week in advance, eliminating contact between experimenter and subject prior to the test. Scheduling in this manner provided two similar groups; in lieu of more appropriate labels one was termed the "Formal" Group and the other the "Informal" Group according to the treatment they would receive.

As indicated, differentiation between the groups was accomplished by the experimenter's attitude toward the subjects and was based upon the degree of formality with which the test was conducted, The so-called
"Formal" Group was processed in a manner similar to that employed in the usual experiments on pain. Little explanation of the test procedure or the apparatus was proffered. Actual testing was conducted in a darkened room surrounded by complex electronic equipment. From the time the subject was called to the testing room to the time he was dismissed, the relations between experimenter and subject were strictly impersonal but not unfriendly. In this group electric shocks were administered by the experimenter after a warning light was flashed.

In the treatment of the "Informal" Group a very casual manner was adopted. This consisted of conversing with the subject in the waiting room, describing as much as was feasible of the experiment without divulging its purpose, explaining the operation of the apparatus, having the light on in the testing room during the explanation, demonstrating the method of shocking (the experimenter applying the electrodes and shocking himself), offering the subject cigarettes, and generally attempting to create an atmosphere of congeniality. In this group electric shocks were self-administered by the subject, after a warning light was flashed.

The groups were organized as follows: "Formal" Group with morphine medication (15 mg.), 16 subjects; "Formal" Group with placebo medication (7.5 mg. thiamin hydrochloride 1 dissolved in a volume of distilled water equal to that of the morphine solution used), six subjects; "Informal" Group with morphine medication, 14 subjects; "Informal" Group with placebo medication, six subjects.

Apparatus and procedure. In another study, which has been described in the preceding paper (10), an apparatus was developed to deliver painful electrical stimuli to the fingers of one hand. The general features of this apparatus will be outlined below. In this study it was determined that verbal reports on the intensities of shock stimuli correlated best with wattage indices, rather than those of voltage or amperage. In other words, insofar as the physical aspects were concerned, the effectiveness of the stimulus in evoking a psychological response was determined by its power (wattage) characteristics. Therefore, the effective strengths of all stimuli in these experiments were controlled on the basis of wattage delivered to the subjects. ${ }^{2}$

For present purposes, the general procedure utilized for all subjects may be described as follows. The sub-

1 In the present studies, a solution of thiamin hydrochloride was used instead of distilled water in order to insure a transient "sting" on subcutaneous injection which resembles that produced by morphine solutions.

2 The term "power" as used in these experiments refers to calculations made on the basis of the equation $\mathbf{P}=\mathbf{I}^{2} \mathbf{R}$. As was shown previously (10), such values represent only "apparent" powers since a biological circuit includes not only resistance but capacitance and possibly inductance. However, as has been pointed out (loc. cit.), for low values of resistance such "apparent" power corresponds quite closely to actual power delivered. 
ject washed his hands before being shown into the quiet, air-conditioned room. Both surfaces of the middle three fingers were cleaned with acetone and thoroughly scrubbed with a tooth-brush coated with electrode paste. (This technique greatly reduced inter-individual differences in skin resistance.) The lateral surfaces of the fingers were wiped with acetone and the electrodes were applied, being fastened in place with a rubber band. By means of an alternating current Wheatstone bridge, a cathode ray oscilloscope, and other instruments, the capacitance in the subject's circuit was balanced out and his skin resistance obtained. Balancing the capacitance before obtaining the resistance was deemed necessary since it is known that the functioning of an organic circuit does not parallel that of a non-organic circuit. Because of this fact, during the development of the apparatus and procedure, empirical charts were constructed that showed the relationship between changes in skin resistance and the voltage that would be required for any specific power. By reference to this chart the experimenter could obtain at a glance the voltage necessary for any desired power at any skin resistance. In this manner a family of wattage curves was employed for pre-setting the stimulator at any desired step of the scale. The scale powers, nine in number, were approximately 0.15 watt, diverging in both directions from a standard of approximately 1.65 watts. $^{3}$ This standard power was a definitely painful stimulus when tested by the experimenters upon themselves. Further information on the standards and scale, including errors of prediction, may be found in the preceding report (10).

After a subject's skin resistance had been obtained, six shocks of progressively increasing power were delivered until the standard was reached, and this stimulus was repeated twice. To give the subject practice in comparing stimuli, one complete series of nine shocks was administered in a predetermined random order at approximately 45 -second intervals. 4 In accordance with instructions, the subject responded to each shock by reporting verbally whether it was stronger or weaker than the previously given standards. On completing this series the subject was given four more standard stimuli preceded by the following information: he was told to remember the strength of the standards since, after a rest interval of one hour, his judgment would be required on several more series. The subject was then returned to the waiting room and medication was administered, if scheduled.

After one hour the subject was returned to the experimental room, prepared in the same manner as before, and the skin resistance obtained. Six test series, which were the same as the practice series, were then administered;

3 For the great majority of subjects nine powers were sufficient to obtain a complete series. However, several individuals required an additional step at either end of the scale.

4 To lessen the influence of stimulus order on verbal reports, two predetermined random orders were employed, each order being given to half of each group.
TABLE I

Comparison of group wattage means for the entire test and for separate halves of the test

\begin{tabular}{|c|c|c|c|c|c|c|}
\hline Group & $\begin{array}{c}\text { Control } \\
\text { mean }\end{array}$ & $\begin{array}{l}\text { Mor- } \\
\text { phine } \\
\text { mean }\end{array}$ & $\begin{array}{l}\text { Mean } \\
\text { diff. }\end{array}$ & $\underset{\mathbf{M d}}{\text { Sigma }}$ & $t$ & P* \\
\hline $\begin{array}{l}\text { Entire "Formal" } \\
\text { Entire "Informal" } \\
\text { 1st three series }\end{array}$ & $\begin{array}{l}1.52 \\
1.58 \\
1.53\end{array}$ & $\begin{array}{l}1.65 \\
1.63 \\
1.68\end{array}$ & $\begin{array}{l}0.13 \\
0.05 \\
0.15\end{array}$ & $\begin{array}{l}0.038 \\
0.038 \\
0.042\end{array}$ & $\begin{array}{l}3.42 \\
1.32 \\
3.57\end{array}$ & $\begin{array}{r}<1 \% \\
>20 \% \\
<1 \%\end{array}$ \\
\hline $\begin{array}{l}\text { 1st three series } \\
\text { "Informal" }\end{array}$ & 1.64 & 1.67 & 0.03 & 0.034 & 0.88 & $>30 \%$ \\
\hline $\begin{array}{l}\text { 2nd three series } \\
\text { "Formal" }\end{array}$ & 1.52 & 1.63 & 0.11 & 0.061 & 1.80 & $>5 \%$ \\
\hline $\begin{array}{l}\text { 2nd three series } \\
\text { "Informal" }\end{array}$ & 1.55 & 1.65 & 0.10 & 0.052 & 1.92 & $>5 \%$ \\
\hline
\end{tabular}

* The $P$ column indicates the percentage of such results that would be expected to occur by chance. The 5 per cent level or less is accepted as showing that a significant difference was obtained.

all series were consecutive except that a three-minute rest period was interpolated between the third and fourth. Prior to each of these test series the subject was told to judge each shock with respect to whether it was stronger or weaker than the previously given standards, and he was cautioned not to compare the shocks with each other.

Treatment of data. Two distinct treatments were applied to the data. The first was the simple method of totalling the "stronger" responses for each individual and calculating the significance of the difference between group means by use of the $t$ test (Edwards [11]). The second method involved comparisons of mean powers (the mean power levels that the subjects judged as being equal to the standards). Although the error between predicted power and delivered power was usually small, it appeared to be of sufficient magnitude to prohibit a direct transformation from the mean relative percentages of "stronger" judgments given by the Spearman Summation Method to mean powers for each individual, since several equalization processes would have been necessary. A closer correspondence between the subject's report and the statistical statements seemed to be maintained by the following method: (1) computing for all subjects the relative percentages of "stronger" judgments by the Spearman Summation Method (Woodworth, [12]) ; 5 (2) transforming the mean relative percentages for each group into power indices and obtaining the "50 per cent points" (the point of subjective equality, i.e., the power that was judged as being equal to the standard); (3) calculating the statistical significance of the differences between the means of the various conditions. For purposes of graphic presentation, the mean percentage of "stronger" judgments of each scale step was plotted against the mean power delivered and

- Although the Spearman Summation Method does not weight errors, it does afford a rather accurate means of converting "stronger" judgments into power when the scale steps are not entirely equivalent. When scale steps are equal the method advocated by Davis (13) would seem more appropriate. 
free-hand smoothing gave the usual psychophysical or ogive curve.

Early in the experiment it became evident that changes in a subject's estimate of the intensities of stimuli occurred frequently as the experiment progressed. Therefore, $t$ tests were made of the differences between the "Formal" and "Informal" Groups, not only for the entire six series but also the first and second halves of the experiment separately.

\section{RESULTS}

"Formal" conditions. Table I and Figure 1 present the results obtained on control and morphine days for the "Formal" Group (power calculations). It can be seen that the " 50 per cent" point (that point on the curve which the subjects judged to be equal to the standard) for the control, or non-morphine days, is at a considerably lower power than that of the standard. This indicates much overestimation of the intensity of stimuli. The corresponding point on the curve for morphine days is at the standard power of 1.65 watts. This shows that no constant or mean error occurred and that the administration of analgesic doses of morphine under these conditions resulted in the elimination of overestimation. A $t$ value of
3.42 was obtained for the difference between the means of these two conditions; for 15 degrees of freedom this difference would be expected to occur by chance once in somewhat more than 100 repetitions of the experiment. It is quite evident, then, that morphine reduced the tendency to overestimate intensities of painful stimuli under the testing conditions utilized for the "Formal" Group.

Results obtained on the "Formal" Group which had placebo medication will not be presented graphically. The mean for control days was 1.56 watts, and the mean for placebo days was 1.54 watts, giving a non-significant $t$ of 0.29 when compared by the difference method. As might have been predicted, both of these means approximated the mean for the control days of the "Formal" Group which received morphine on medication days. These results show overestimation of the intensities of painful stimuli and no reduction following the administration of the placebo. ${ }^{\circ}$

6 The inefficacy of placebos in altering the overestimation of intensities of painful stimuli in the "Formal" Group may be related to the particular type of subjects used in these experiments; post-addicts are sufficiently experienced to recognize the nature of the medi-

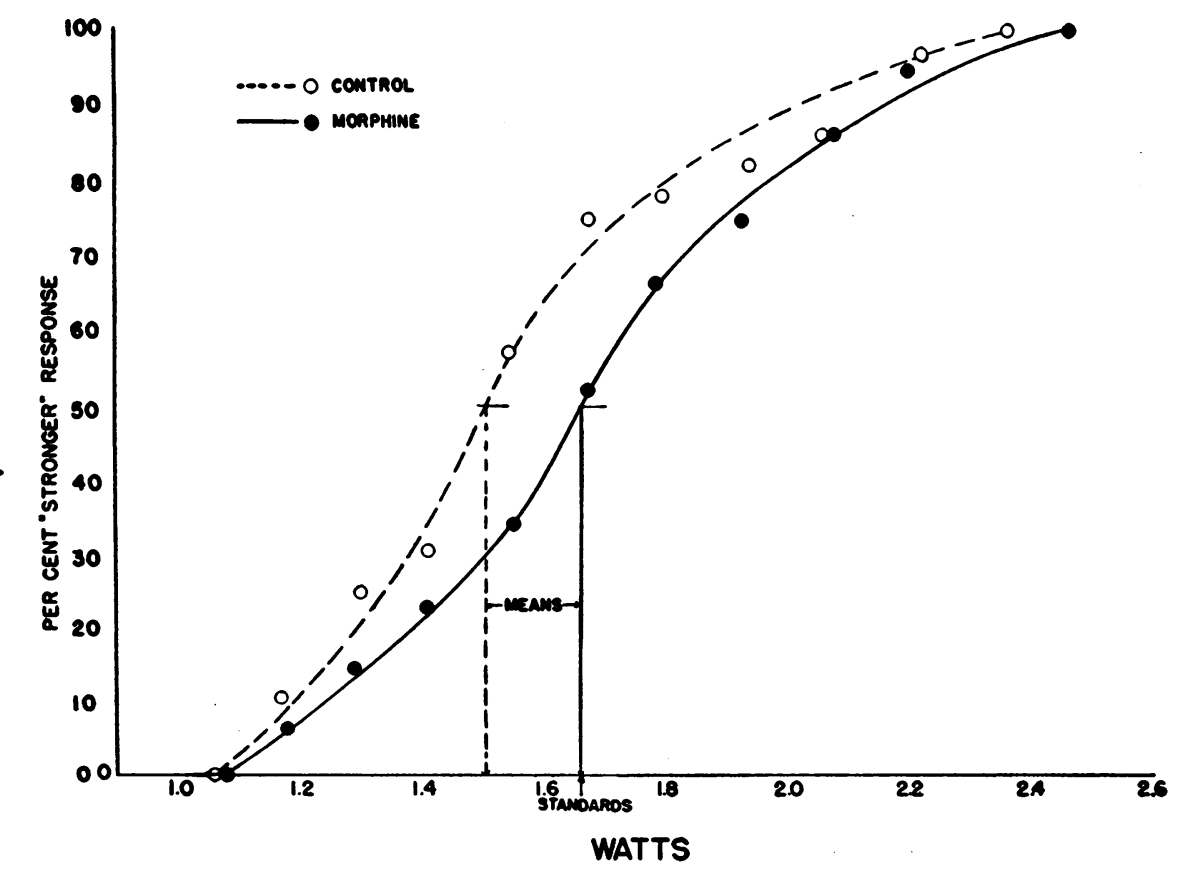

Fig. 1. Frequency of the Response "Stronger" in Delayed Comparison of Electric Shock Stimuli ("Formal" Group, $\mathrm{N}=16$ )

For explanation, see text. 


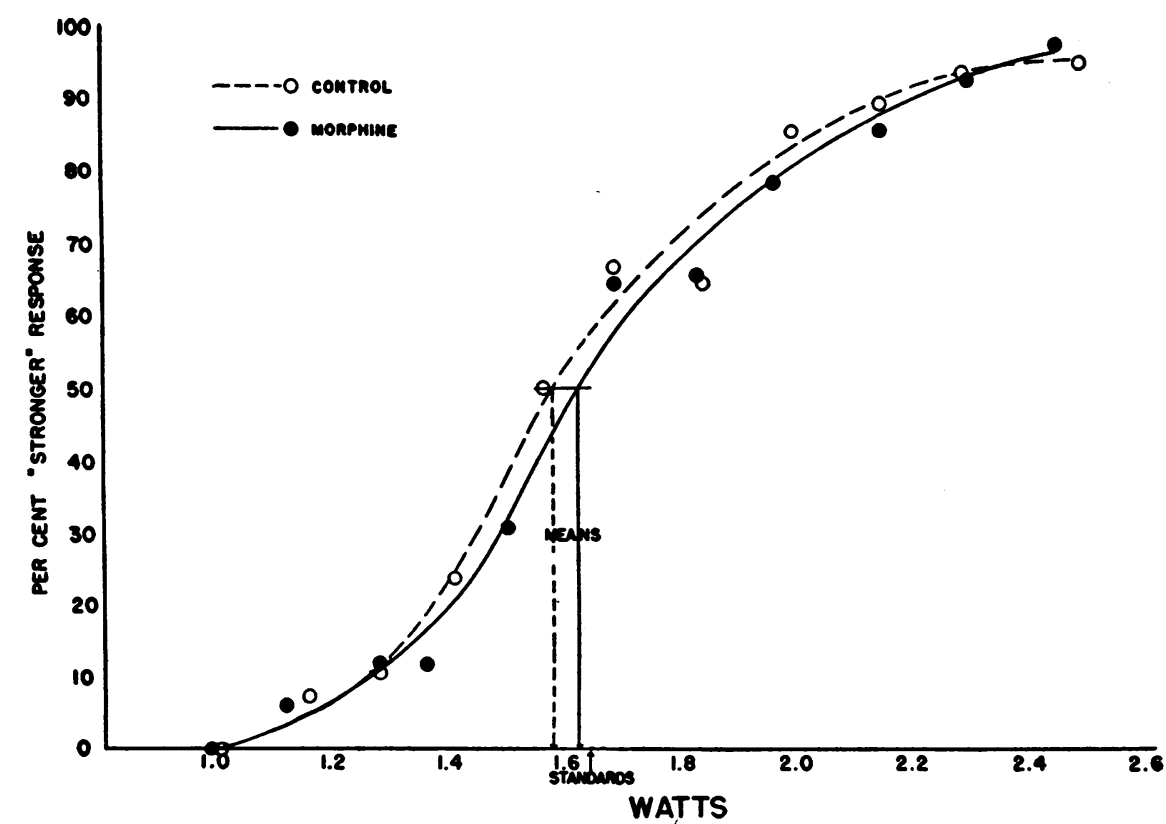

Fig. 2. Frequency of the Response "Stronger" in Delayed Comparison of Electric Shock Stimuli ("Informal" Group, $N=14$ )

For explanation, see text.

"Informal" conditions. Table I and Figure 2 show the results obtained on the "Informal" Group (control, and morphine medication). The "50 per cent point" for control days is somewhat lower than the standard, and the corresponding point for morphine days is also slightly lower than the standard, but approximates it closely. The difference between the means of these two conditions is non-significant. The $t$ of 1.32 for 13 degrees of freedom indicates that this difference would be expected to occur by chance once in four repetitions of the experiment.

As before, the outcome on the placebo group for the informal conditions will not be illustrated. The mean for control days was 1.65 watts, and the mean for placebo dáys was 1.57 watts, giving a non-significant $t$ of 1.14 for the difference between them. It may be seen that the " 50 per cent point" for control days under these informal conditions was at the standard, and that this point for placebo days was at a somewhat lower power level.

Further analysis. In the section on treatment

cation. It might be expected that in non-sophisticated subjects placebos might have different effects (Wolff and Goodell [14]), since suggestion is known to elevate pain perception thresholds, and to relieve clinical pain. of the data it was mentioned that separate statistical treatments had been given the first and second halves of the six test series. The present hypothesis would be strengthened considerably if a greater difference between control days of the "Formal" and "Informal" Groups were shown to exist for the first three series and if a regression toward more common judgments occurred toward the end of the experiment. This reasoning is based on the assumption that, as the experiment continued, the increasing familiarity of the "Formal" Group with the shock and the general experimental situation would tend to diminish their anxiety. On the other hand, repeated painful shock stimuli might be expected to develop some anxiety in the "Informal" Group.

Table II presents the data on total "stronger" responses for the entire experiment and for the separate halves of the experiment. The first two comparisons, those between sums of "stronger" responses for the entire six series, show that morphine significantly reduced the total number of "stronger" judgments for both groups. After separating the data into first and second halves, it was found that morphine reduced significantly the number of "stronger" judgments for the "For- 
TABLE II

Comparison of "stronger" responses for the entire test and for separate halves of the test

\begin{tabular}{|c|c|c|c|c|c|c|}
\hline Group & $\begin{array}{c}\text { Control } \\
\text { mean }\end{array}$ & $\begin{array}{l}\text { Mor- } \\
\text { phine } \\
\text { mean }\end{array}$ & $\begin{array}{c}\text { Mean } \\
\text { diff. }\end{array}$ & $\underset{\mathbf{M d}}{\text { Sigma }}$ & $t$ & $\mathbf{P}$ \\
\hline $\begin{array}{l}\text { Entire "Formal" } \\
\text { Entire "Informal" } \\
\text { 18t three series } \\
\text { "Formal" } \\
\text { 18t three series } \\
\text { "Informal" } \\
\text { 2nd three series } \\
\text { "Formal" } \\
\text { 2nd three series } \\
\text { "Informal" }\end{array}$ & $\begin{array}{l}32.50 \\
29.57 \\
16.50 \\
13.78 \\
16.00 \\
15.79\end{array}$ & $\begin{array}{l}26.94 \\
26.79 \\
13.13 \\
13.21 \\
13.81 \\
13.71\end{array}$ & $\begin{array}{l}5.56 \\
2.79 \\
3.37 \\
0.57 \\
2.19 \\
2.08\end{array}$ & $\begin{array}{l}1.98 \\
1.23 \\
1.06 \\
0.67 \\
1.48 \\
1.04\end{array}$ & $\begin{array}{l}2.81 \\
2.27 \\
3.18 \\
0.85 \\
1.48 \\
2.00\end{array}$ & $\begin{array}{r}<2 \% \\
<5 \% \\
<1 \% \\
>40 \% \\
>10 \% \\
>5 \%\end{array}$ \\
\hline
\end{tabular}

mal" Group in the first three series $(P<1)$, but had no such effect upon the "Informal" Group $(P>40)$. The difference between the groups is at the 5 per cent level of confidence. These findings show that the experimental procedure was effective and that morphine exerted a significant reduction of overestimation in only the "Formal" Group.

A different outcome occurred in the second halves of the experiment. Although morphine lowered the number of "stronger" responses in both groups the reduction approached significance only in the "Informal" Group. The difference in total "stronger" responses in the last three series on control days between the "Formal" and "Informal" Groups was not significant $(P>90)$. This shows a trend toward common judgments.

Similar outcomes are shown to have occurred when calculated power means of the separate halves of the experiment are compared, (Table I). (In this connection, it should be remembered that an overestimation of stimuli is inferred when a lower power is estimated as being equal to the standard, $c f$. Figure 1.) As mentioned, the administration of morphine resulted in a significant reduction in the overestimation of the "Formal" Group for the entire six series, but resulted in a slight, non-significant reduction in the estimation of the "Informal" Group. A much greater difference in favor of our hypothesis is observed to occur when the mean powers (mean estimation in terms of watts) of only the first three series are compared. Table I shows that the difference between the control and morphine means of the "Formal" Group was significant at less than the 1 per cent level, and that the difference for the "Informal" Group was non-significant, being greater than the 30 per cent level of chance oc- currence. This comparison shows again the very strong influence of the experimental procedure. In the comparisons of mean powers of the second halves of the experiment, the data indicate that the groups approached a more common basis of judgment. This is in agreement with calculation of total "stronger" responses during the same period.

\section{DISCUSSION}

Both methods of evaluating the data yielded unequivocal results. Computations based upon total "stronger" responses and those based upon mean powers were in close agreement. These data indicate that when the experiment was conducted under informal conditions, pain intensities were overestimated only slightly and morphine had no significant effect, if any, on discrimination. In contrast, marked overestimations of pain intensities occurred under formal control conditions and morphine practically abolished this error. In effect, therefore, morphine enabled the "anxious" groups to estimate the intensities of painful stimuli as well as the group in which anxiety had been dissipated. That this effect was due to a specific action of morphine is indicated by the fact that administration of placebos had no significant effect upon the estimation of painful stimuli in either group.

From these results it may be inferred that ordinary formal experimental conditions tend to produce anxiety which promotes overestimation of the intensities of painful stimuli. The effects of morphine, as shown in these experiments, therefore, can be interpreted as due to a reduction of anxiety.

Furthermore, it has been shown that morphine tends to relieve anxiety which is produced by two different types of experimental situations. Considering the results for the first half of the test for the "Formal" Group, it was shown that anticipatory reactions to the unknown aspects of the experimental situation resulted in significant overestimation of pain intensities and that morphine significantly reduced this overestimation. It may be inferred that in this group anxiety lacked a clear and definite object. However, it was shown also that overestimation was induced in the "Informal" Group during the second half of the test, and that administration of morphine resulted in a reduction of this error, which was close 
to being significant. The overestimation of this group seemed to be due to previously applied shock. Both of these types of anxiety, namely, with and without a definite object, were relieved by morphine.

However, there is evidence that not all types of anxiety are reduced by morphine. For example, in subjects who exhibited suspicion and hostility toward the experimenters, morphine had little effect on the overestimation of intensities of painful stimuli. Therefore, it would appear that although morphine reduces anticipatory fear of pain, it may not act as a general anxiety relieving agent. This inference is supported by data obtained from a psychodynamic study of an addicted patient by Wikler (15) which indicate that morphine reduces such anxiety as is related to inadequate gratification of so-called "primary" needs, such as hunger, fear of pain and sexual (general erotic) urges, while the drug has little effect on such anxiety as is related to inadequate gratification of "secondary" needs, such as narcissism, dependence, etc.

That "emotional factors" may influence pain threshold measurements and the effects of drugs thereon has been suggested and demonstrated by several investigators; this aspect has been discussed in the review by Wikler (1). Judging from our present studies, it appears that anticipatory fear of pain is one such important variable which must be controlled in investigations of this sort. Failure to do so may account, in part, for the wide discrepancies which have been reported by different observers in such investigations.

Because of its considerable theoretical importance in relation to the problem of pain, a more direct method for quantitative measurement of the effects of anticipatory fear of pain has been devised. This will be described in detail in another communication (16).

\section{SUM MARY AND CONCLUSIONS}

1. The effects of subcutaneous injections of 15 mg. of morphine on the ability of subjects (postaddicts) to judge the intensity of painful electric shock stimuli were studied under two conditions: (a) under formal conditions, proceeding with the experiment without familiarizing the subjects with the potentially fear-inspiring experimental situa- tion; (b) under informal conditions, preceding the experiment with reassurance, demonstration and explanation designed to allay the subject's anxiety. In both groups six consecutive series of nine stimuli were delivered in each experiment, and the subjects were required to state whether each stimulus was "stronger" or weaker than a standard stimulus. The strength of each stimulus was controlled on the basis of the power (wattage) value delivered to the subject.

2. During the first three series under formal control conditions, a significantly greater number of test stimuli were judged "stronger" in comparison with the standard stimuli than were actually delivered, and the power (wattage) values of the test stimuli at the points of subjective equality ( 50 per cent point) were lower than those of the standard stimuli. These errors were reduced significantly by administration of morphine, but were not altered by placebos.

Under informal control conditions, verbally reported judgments of test stimuli were extremely accurate and power values of the test stimuli at the points of subjective equality were practically identical with those of the standards. Neither morphine nor placebos had any significant effect on the estimation of the intensities of painful stimuli.

3. Under control conditions during the last three series the accuracy of estimation of the intensities of painful stimuli remained practically unchanged in the "Formal" Group but deteriorated in the "Informal" Group. In both groups morphine tended to reduce the magnitude of error, while placebos had no apparent effect.

4. It is concluded that (a) under conditions which promote anxiety or fear of pain, subjects tend to overestimate the intensities of painful stimuli; (b) morphine reduces such anxiety; (c) under conditions in which anxiety is largely eliminated, little if any overestimation of the intensities of painful stimuli occurs; $(d)$ morphine does not affect the ability of subjects to accurately estimate the intensities of painful stimuli when anxiety is dissipated; and $(e)$ anxiety, particularly that which is associated with anticipation of pain, is one important variable which must be controlled in experimental investigations of problems related to pain and analgesia. 


\section{REFERENCES}

1. Wikler, A., Sites and mechanism of action of morphine and related drugs in the central nervous system. Pharmacol. Rev., 1950, 2, 435.

2. Edwards, W., Recent research on pain perception. Psychol. Bull., 1950, 47, 449.

3. Wolff, H. G., and Wolf, S., Pain. Charles C Thomas, Springfield, Ill., 1948.

4. Wolff, H. G., Hardy, J. D., and Goodell, H., Studies on pain. Measurement of the effect of morphine, codeine, and other opiates on the pain threshold and an analysis of their relation to the pain experience. J. Clin. Invest., 1940, 19, 659.

5. Andrews, H. L., The effects of opiates on the pain thresholds in post-addicts. J. Clin. Invest., 1943, 22, 511.

6. Isbell, $H$., Unpublished data.

7. Denton, J. E., and Beecher, H. K., New analgesics. J. A. M. A., 1949, 141, 1051.

8. Chapman, W. P., and Jones, C. M., Variations in cutaneous and visceral pain sensitivity in normal subjects. J. Clin. Invest., 1944, 23, 81.

9. Isbell, H., and Frank, C., Unpublished data.
10. Hill, H. E., Flanary, H. G., Kornetsky, C. H., and Wikler, A., Relationship of electrically induced pain to the amperage and the wattage of shock stimuli. J. Clin. Invest., 1952, 31, 464.

11. Edwards, A. L., Statistical Analysis for Students in Psychology and Education. Rinehart \& Co., Inc., New York, 1946, p. 174.

12. Woodworth, R. S., Experimental Psychology. Henry Holt \& Co., Inc., New York, 1939, p. 402.

13. Davis, R. C., The comparison of series of proportions : the problem and a new solution. Psychol. Bull., 1951, 48, 348.

14. Wolff, H. G., and Goodell, H., The relation of attitude and suggestion to the perception of and reaction to pain. A. Research Nerv. \& Ment. Dis., Proc., 1943, 23, 434.

15. Wikler, A., A psychodynamic study of a patient during self-regulated re-addiction to morphine. Psychiat. Quart., to be published.

16. Hill, H. E., Kornetsky, C. H., Flanary, H. G., and Wikler, A., Studies on anxiety associated with anticipation of pain. I. Effects of morphine. Arch. Neurol. \& Psychiat., to be published. 